\title{
Retrospective study of the combined application of the blink reflex and lateral spread reflex in microvascular decompression of facial nerve surgery
}

\author{
Yang Zhang^, Lihui Xia^^, Jingzhi Lin^, Yong Zhang \\ Department of Neurosurgery, Guangdong Second Provincial General Hospital, Guangzhou, China \\ Contributions: (I) Conception and design: Yang Zhang, Yong Zhang; (II) Administrative support: Yang Zhang, Yong Zhang; (III) Provision of study \\ materials or patients: Yang Zhang, L Xia; (IV) Collection and assembly of data: L Xia, J Lin; (V) Data analysis and interpretation: L Xia, J Lin; (VI) \\ Manuscript writing: All authors; (VII) Final approval of manuscript: All authors. \\ Correspondence to: Yong Zhang. Department of Neurosurgery, Guangdong Second Provincial General Hospital, 466 Xingangzhong Road, Haizhu \\ District, Guangzhou 510317, China. Email: zhangyongsey@163.com.
}

Background: The blink reflex (BR) can be used as a routine monitoring method during facial nerve
microvascular decompression. This study aimed to investigate whether the use of the BR in hemifacial spasm
(HFS) surgery is comparable to that of the lateral spread reflex (LSR), and to explore its significance for
guiding intraoperative neurophysiological monitoring (IONM).
Methods: Patients undergoing facial nerve microvascular decompression from 2016 to 2018 were included
in the study. According to the results of IONM, the intraoperative monitoring items of the BR and two
conventional facial nerve microvascular decompression procedures, namely the marginal mandibular branch
LSR (MAR-LSR) and zygomatic branch LSR (ZYG-LSR), were compared. We mainly compared whether
there were differences in the occurrence rate, disappearance rate, waveform, occurrence current, and
prognosis of the three monitoring methods. Results: The occurrence rate of the BR was lower than that of the MAR-LSR and ZYG-LSR, as well as the three combined detection groups. The disappearance rate of the BR was not different to that of the MARLSR, but higher than that of the ZYG-LSR group. In addition, the waveform of the BR showed differences from that of the MAR-LSR and ZYG-LSR. The incidence of postoperative residual symptoms in patients with any kind of reflex on the first day after surgery and the day of discharge was significantly higher than that of patients in which all three reflexes disappeared.

Conclusions: Combined BR and LSR monitoring can reduce the occurrence of postoperative residual symptoms. We suggest that by increasing the use of BR examination during surgery, the integrity of the trigeminal nerve can be protected.

Koywords: Blink reflex (BR); lateral spread reflex (LSR); intraoperative neurophysiological monitoring (IONM); hemifacial spasm (HFS); abnormal muscle response (AMR)

Submitted Sep 30, 2021. Accepted for publication Dec 10, 2021.

doi: 10.21037/apm-21-3197

View this article at: https://dx.doi.org/10.21037/apm-21-3197

^ ORCID: Yang Zhang, 0000-0002-6333-9488; Lihui Xia, 0000-0002-4560-695X; Jingzhi Lin, 0000-0003-2563-1442. 


\section{Introduction}

According to the consensus among Chinese experts (1), neurophysiological monitoring technologies which are commonly used during the perioperative period of facial nerve microvascular decompression include brainstem auditory evoked potential (BAEP), somatosensory evoked potential (SSEP), resting electromyography, abnormal muscle response (AMR), and Z-L response. This work has provided a guideline which has been approved by some scholars $(1,2)$. In clinical practice, the blink reflex (BR) is also an effective method which is commonly used for facial nerve monitoring $(3,4)$. Since intravenous anesthesia during surgery affects the BR, there is a lack of information about its use in intraoperative electrophysiological monitoring.

Patients with hemifacial spasm (HFS) generally undergo various neurophysiological examinations, including the BR and/or lateral spread reflex (LSR) in the awake state before or after surgery and LSR monitoring during surgery. The LSR is especially widely used since it can directly reveal whether specific blood vessels supplying the facial nerve are relieved of compression $(1,3,4)$. However, in practice, the use of the LSR may have multiple complications. These include: (I) the LSR cannot be elicited before surgery; (II) the LSR disappears before reaching the decompression site during the surgery; (III) the LSR does not disappear after apparently sufficient decompression (5-7).

In this study, we combined the marginal mandibular branch LSR (MAR-LSR), zygomatic branch LSR (ZYGLSR), and BR to monitor facial nerve compression, and comprehensively evaluated whether the decompression was sufficient. We present the following article in accordance with the STROBE reporting checklist (available at https:// dx.doi.org/10.21037/apm-21-3197).

\section{Methods}

All procedures performed in this study involving human participants were in accordance with the Declaration of Helsinki (as revised in 2013). This study was approved by the Medical Ethics Committee of Guangdong Second Provincial General Hospital (DG2H-KY IRBAFSC.10-01.1). All patients provided their consent for inclusion in the study.

\section{Subjects}

From March 2016 to September 2018, 257 patients underwent facial nerve microvascular decompression. All patients had been diagnosed with primary HFS, and were undergoing facial nerve microvascular decompression for the first time. A total of 236 patients underwent three monitoring examinations at the same time, including the MAR-LSR, ZYG-LSR, and BR (patients not enrolled included 6 patients who underwent secondary surgery, 1 tumor patient, and 14 others).

\section{Recording methods}

Electrophysiological recordings were performed using standard techniques of electrode needle recording (Xi'an Friendship Medical Electronics Co., Ltd., Xi'an, China). The intraoperative neuromonitoring device used was the Cascade Elite (Cadwell Industries, USA).

\section{Intraoperative neurophysiological monitoring (IONM) procedure}

The IONM steps mainly refer to the research of Koht et al. (8). During microvascular decompression in patients with HFS, when the effect of muscle relaxant induced by anesthesia decreases, the AMR of orbicularis oculi muscle can be obtained by stimulating the marginal mandibular branch, the AMR of orbicularis oris muscle can be obtained by stimulating the zygomatic branch of facial nerve, and the AMR of orbicularis oris muscle can be obtained by stimulating the supraorbital nerve.

\section{Anesthesia}

Anesthesia was induced using sufentanil, propofol, and a single-dose, short-term muscle relaxant (rocuronium; $0.2 \mathrm{mg} / \mathrm{kg}$ ), and general anesthesia was performed using continuous intravenous infusion of remifentanil and propofol.

\section{BR (Figure 1A)}

Analysis window: $100 \mathrm{~ms}, 10 \mathrm{~ms} / \mathrm{Div}, 100 \mu \mathrm{V} / \mathrm{Div}$. Filter settings: $3,000 \mathrm{~Hz}$ high cut-off, $20 \mathrm{~Hz}$ low cut-off, $50 \mu \mathrm{V}$ gain. Recoding sites: bilateral orbicularis oculi muscle (direct motor potential), orbicularis oris muscle (abnormal motor potential) of the affected side. Stimulation parameters: single stimulation, $100-200 \mu$ s pulse width, $10-30 \mathrm{~mA}$. The active electrode was placed above the supraorbital fissure.

\section{MAR-LSR (Figure 1B)}

Analysis window: $50 \mathrm{~ms}, 5 \mathrm{~ms} / \mathrm{Div}, 100 \mu \mathrm{V} / \mathrm{Div}$. Filter 

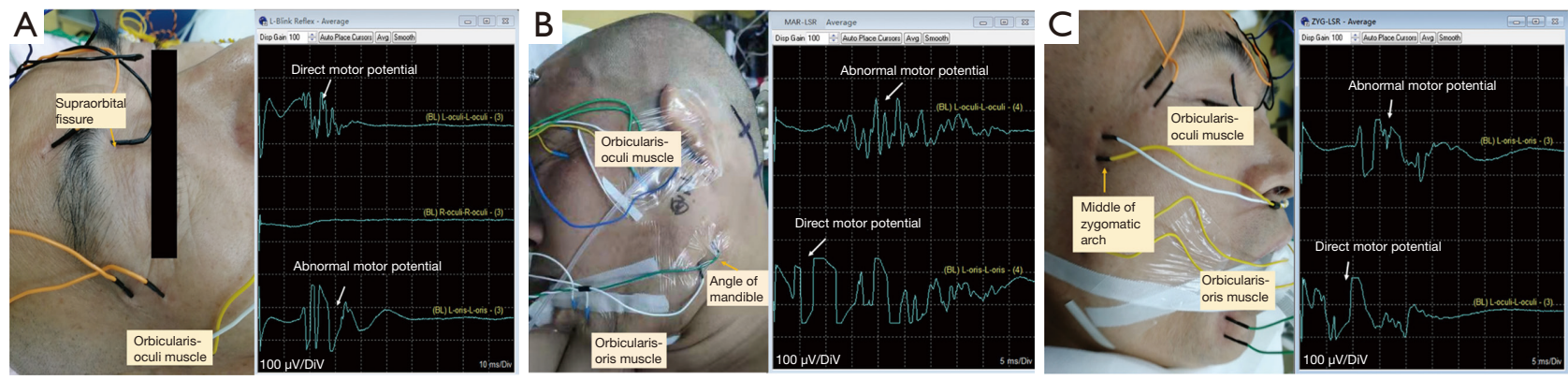

Figure 1 Intraoperative electrode arrangement and monitoring window. (A) BR; (B) MAR-LSR; (C) ZYG-LSR. BR, blink reflex; MARLSR, marginal mandibular branch lateral spread reflex; ZYG-LSR, zygomatic branch lateral spread reflex.

settings: 3,000 Hz high cut-off, $20 \mathrm{~Hz}$ low cut-off, $50 \mu \mathrm{V}$ gain. Recoding sites: orbicularis oculi muscle (abnormal motor potential), orbicularis oris muscle (direct motor potential) of the affected side. Stimulation parameters: single stimulation, $100-200 \mu$ s pulse width, $5-30 \mathrm{~mA}$. The active electrode was placed at the angle of the mandible.

\section{ZYG-LSR (Figure 1C)}

Analysis window: $50 \mathrm{~ms}, 5 \mathrm{~ms} / \mathrm{Div}, 100 \mu \mathrm{V} / \mathrm{Div}$. Filter settings: $3,000 \mathrm{~Hz}$ high cut-off, $20 \mathrm{~Hz}$ low cut-off, $50 \mu \mathrm{V}$ gain. Recoding sites: orbicularis oculi muscle (direct motor potential), orbicularis oris muscle (abnormal motor potential) of the affected side. Stimulation parameters: single stimulation, $100-200 \mu$ s pulse width, 5-30 mA. The active electrode was placed in the middle of the zygomatic arch.

\section{Monitoring indicators}

From the intraoperative monitoring data, the stimulation intensity, latency, amplitude of direct and abnormal motor potentials, and the disappearance status of abnormal motor potentials from the three examinations (MAR-LSR, ZYGLSR, and BR) were obtained. According to the consensus of experts on microvascular decompression, we compared the similarities and differences of the three stimulation methods, as well as their effects on prognosis.

\section{Statistical analyses}

The data was analyzed using SPSS 24.0 statistical software. Normally distributed data are expressed as mean \pm standard deviation (SD) and were analyzed by Student's $t$-test. The skewness of the data are represented by the median and quartile $M\left(Q_{25}, Q_{75}\right)$, and analyzed by the normal approximation method of the Mann-Whitney $U$ test. The classification data is expressed as the number of cases and percentile $\mathrm{n}(\%)$. Pearson's chi-squared test was used when $n \geq 40$ and theoretical frequency $T \geq 5$. Fisher's exact probability method was used when $\mathrm{n}<40$ or $\mathrm{T}<1$. Fisher's exact probability method was used when theoretical frequency $\mathrm{T}<1$ or $\mathrm{T}<5$ in the multi-classification data exceeded $1 / 5$ th of the total number of theoretical frequencies. If there was a statistical difference between the groups, further pairwise comparisons were made. $\mathrm{P}<0.05$ indicates statistical significance.

\section{Results}

\section{General information}

The mean age of the 236 patients included in the study was $48.79 \pm 29.21$ years, and the ages ranged from 25 to 78 years. The ratio of males to females was $73: 163$. In this cohort, the affected side showed no advantage. The Cohen scores were grade II-IV. The median onset period was 5.78 [0.3-30] years. The median surgery time was 172.29 [100-270] mins, and the median hospitalization time was 10.13 [5-23] days. The offending vessel was identified in the 236 patients enrolled in the study and the 6 patients undergoing secondary surgery. The responsible vessels were judged by the main surgeon and verified by intraoperative electrophysiological monitoring (Table 1). The general data of the patients included in this study are similar to those reported in the literature (6,9-12). Complications in the study are mostly delayed facial palsy (incidence $10.71 \%$ ), hearing loss (incidence $2.67 \%$ ), dizziness (incidence $10.71 \%$ ), headache (incidence $4.29 \%$ ), and wound infection (incidence $0.83 \%$ ). Delayed facial palsy and hearing loss will be treated with steroids and the rest of the cases will be 
treated symptomatically.

\section{Occurrence rate: BR compared with $M A R-L S R$ and ZYG-LSR}

According to different monitoring methods, three groups of monitoring results (BR, MAR-LSR, and ZYG-LSR) could be obtained. At first, we compared the occurrence

Table 1 Characteristics of patients with HFS $(n=236)$

\begin{tabular}{lc}
\hline Characteristics & $\mathrm{N}(\%) /$ median [range] \\
\hline Age (years) & $48[25-78]$ \\
Sex (F:M) & $163(69.7): 73(30.93)$ \\
Affected side (Lt:Rt) & $123(52.12): 113(47.88)$ \\
Duration of symptoms (years) & $5.78[0.3-30]$ \\
Operation time duration (mins) & $172.29[100-270]$ \\
Hospitalization time (days) & $10.13[5-23]$ \\
Offending vessel & \\
AICA & $210(86.78)$ \\
PICA & $20(8.26)$ \\
SCA & $3(1.24)$ \\
VEIN & $1(0.41)$ \\
VA & $3(1.24)$ \\
VA + AICA & $3(1.24)$ \\
VA + PICA & $2(0.83)$ \\
\hline
\end{tabular}

HFS, hemifacial spasm; F, female; M, male; Lt, left; Rt, right; AICA, anterior inferior cerebellar artery; PICA, posterior inferior cerebellar artery; SCA, superior cerebellar artery; VEIN, petrosal vein; VA, vertebral artery. rate of the BR group with the MAR-LSR and ZYG-LSR group. The results in Table 2 showed that the occurrence rates of the MAR-LSR $\left(\chi^{2}=23.516 ; \mathrm{P}<0.001\right)$ and ZYG$\operatorname{LSR}\left(\chi^{2}=18.778 ; \mathrm{P}<0.001\right)$ were significantly higher than the occurrence rate of the BR.

The three types of monitoring results were mainly divided into two outcomes: occurrence and non-occurrence. The following four situations indicated "occurrence": all three methods occurrence; "BR" and "MAR-LSR" occurrence, "ZYG-LSR" non-occurrence; "BR" and "ZYG-LSR" occurrence, "MAR-LSR" non-occurrence; "MAR-LSR" and "ZYG-LSR" occurrence, and "BR" nonoccurrence. The occurrence rate was $97.46 \%(n=230)$. The following four cases indicated "non-occurrence": "MAR-LSR" and "ZYG-LSR" non-occurrence, but "BR" occurrence; "BR" and "MAR-LSR" non-occurrence, and "ZYG-LSR" occurrence; "BR" and "ZYG-LSR" nonoccurrence, "MAR-LSR" occurrence; all three methods non-occurrence. The non-occurrence rate was $2.54 \%(n=6)$. The occurrence rate of combined detection was higher than that of the BR, and the difference was statistically significant $(\mathrm{P}<0.001)$. However, the difference in occurrence rate between the combined detection and MAR-LSR $(\mathrm{P}=1.000)$ and ZYG-LSR ( $\mathrm{P}=0.727)$ was not statistically significant.

In summary, the occurrence rate of the BR was lower than that of the MAR-LSR or ZYG-LSR, and the occurrence rate of combined testing was also higher than the BR group.

\section{Disappearance rate: BR compared with MAR-LSR and ZYG-LSR}

There was no significant difference in the disappearance rate between the BR and MAR-LSR groups $\left(\chi^{2}=2.272\right.$;

Table 2 Differences in the occurrence rate of the BR and MAR-LSR or ZYG-LSR

\begin{tabular}{|c|c|c|c|c|c|}
\hline \multirow{2}{*}{ Outcome } & \multirow{2}{*}{$\mathrm{N}$} & \multicolumn{2}{|c|}{$\mathrm{BR}(\mathrm{n}=236)$} & \multirow{2}{*}{$\chi^{2}$} & \multirow{2}{*}{$\mathrm{P}$} \\
\hline & & Occurrence $(n=202)$ & Non-occurrence ( $n=34)$ & & \\
\hline MAR-LSR, n (\%) & & & & 23.516 & $<0.001$ \\
\hline Occurrence & 229 & 200 (99.01) & 29 (85.29) & & \\
\hline Non-occurrence & 7 & $2(0.99)$ & $5(14.71)$ & & \\
\hline ZYG-LSR, n (\%) & & & & 18.778 & $<0.001$ \\
\hline Occurrence & 228 & 197 (97.52) & 31 (91.18) & & \\
\hline Non-occurrence & 8 & $5(2.48)$ & $3(8.82)$ & & \\
\hline
\end{tabular}

BR, blink reflex; MAR-LSR, marginal mandibular branch lateral spread reflex; ZYG-LSR, zygomatic branch lateral spread reflex. 
Table 3 BR $v s$. MAR-LSR latency and amplitude difference

\begin{tabular}{|c|c|c|c|c|c|}
\hline Waveform & $\mathrm{N}$ & \multicolumn{2}{|c|}{ Group } & Z & $\mathrm{P}$ \\
\hline \multicolumn{6}{|c|}{ Direct motor potential, $M\left(Q_{25}, Q_{75}\right)$} \\
\hline Latency (ms) & 420 & $12.47(11.54,14.14)$ & $2.76(2.29,3.17)$ & -17.647 & $<0.001$ \\
\hline Amplitude (mV) & 419 & $115.26(71.97,169.27)$ & $200.00(199.06,200.63)$ & -18.523 & $<0.001$ \\
\hline Latency (ms) & 432 & $13.02(12.04,14.66)$ & $8.38(7.63,9.15)$ & -17.658 & $<0.001$ \\
\hline Amplitude (mV) & 432 & $157.51(73.99,196.30)$ & $153.61(94.13,188.18)$ & -17.614 & $<0.001$ \\
\hline
\end{tabular}

Table 4 BR $v s$. ZYG-LSR latency and amplitude difference

\begin{tabular}{|c|c|c|c|c|c|}
\hline Waveform & $\mathrm{N}$ & \multicolumn{2}{|c|}{ Group } & Z & $\mathrm{P}$ \\
\hline \multicolumn{6}{|c|}{ Direct motor potential, $M\left(Q_{25}, Q_{75}\right)$} \\
\hline Latency (ms) & 419 & $12.47(11.54,14.14)$ & $2.76(2.29,3.17)$ & -17.342 & $<0.001$ \\
\hline Amplitude (mV) & 418 & $115.26(71.97,169.27)$ & $200.00(199.06,200.63)$ & -18.301 & $<0.001$ \\
\hline Latency (ms) & 430 & $13.02(12.04,14.66)$ & $8.38(7.63,9.15)$ & -18.355 & $<0.001$ \\
\hline Amplitude (mV) & 430 & $157.51(73.99,196.30)$ & $153.61(94.13,188.18)$ & -18.490 & $<0.001$ \\
\hline
\end{tabular}

$\mathrm{M}\left(\mathrm{Q}_{25}, \mathrm{Q}_{75}\right)$ : median and quartile (quarter, three-quarter). BR, blink reflex; ZYG-LSR, zygomatic branch lateral spread reflex.

$\mathrm{P}=0.132)$. However, the disappearance rate of the BR group was higher than that of the ZYG-LSR group $\left(\chi^{2}=7.364\right.$; $\mathrm{P}=0.007)$. These results suggested that the disappearance rate of the BR was similar to that of the MAR-LSR, whilst the difference between the BR and ZYG-LSR was statistically significant.

\section{Waveform differences between BR and MAR-LSR or ZYG-LSR}

We assessed differences in the latency and amplitude of the waveform of BR. Normality tests showed that the latency and the amplitude of the direct motor potential, as well as the latency of the abnormal motor potential, did not conform to a normal distribution. The rank sum test was then applied.

The results of Table 3 showed that the latency of the direct motor potential $(Z=-17.647 ; \mathrm{P}<0.001)$ and the abnormal motor potential $(Z=-17.658 ; \mathrm{P}<0.001)$ were longer in the BR group than in the MAR-LSR group. The amplitudes of the direct motor potential $(Z=-18.523$; $\mathrm{P}<0.001)$ were shorter in the BR group than in the MARLSR group. However, the amplitudes of abnormal motor potential in the BR group showed the opposite result. In Table 4, the changes in latency and amplitudes of the direct and abnormal motor potential in the ZYG-LSR group were similar to the results of the MAR-LSR group.

\section{Differences in current between $B R, M A R-L S R$, and ZYG- LSR}

After testing the data for normality, the Pearson chi-square test was used to assess stimulation current. No statistical differences were found in the stimulation current between the three groups, suggesting that the average extraction current is operatively similar. The average current of the BR was $14.91 \mathrm{~mA}$, MAR-LSR was $13.27 \mathrm{~mA}$, and ZYGLSR was $13.5 \mathrm{~mA}$. 
Table 5 Comparison of postoperative residual symptoms

\begin{tabular}{|c|c|c|c|c|c|c|}
\hline Symptom & $\mathrm{N}$ & \multicolumn{4}{|c|}{ Group } & $P$ \\
\hline First day after operation, n (\%) & & & & & & $<0.001$ \\
\hline Persistent & 13 & $2(22.22)^{d}$ & $1(14.29)$ & $3(42.86)^{d}$ & $7(3.20)$ & \\
\hline Disappeared & 229 & 7 (77.78) & $6(85.71)$ & $4(57.14)$ & $212(96.80)$ & \\
\hline Persistent & 10 & $1(11.11)$ & $1(14.29)$ & $3(42.86)^{d}$ & $5(2.28)$ & \\
\hline Disappeared & 232 & $8(88.89)$ & $6(85.71)$ & $4(57.14)$ & $214(97.72)$ & \\
\hline
\end{tabular}

Examinations were performed for the MAR-LSR, ZYG-LSR, or BR. Group A: symptoms persisted for a single monitoring examination; group B: symptoms persisted for two monitoring examinations; group C: symptoms persisted for all three examinations; group D: none persisted. "P" is group A, B, C vs. group D, $P<0.001$. $^{\text {d }}$, compared with group D. MAR-LSR, marginal mandibular branch lateral spread reflex; ZYG-LSR, zygomatic branch lateral spread reflex; BR, blink reflex.

\section{Disappearance of BR, MAR-LSR, and ZYG-LSR, and short-term prognosis}

As shown in Table 5, the results showed that the occurrence rates of postoperative residual symptoms on the first day after surgery $(\mathrm{P}<0.001)$ and on the day of discharge $(\mathrm{P}<0.001)$ were different between the four groups. That's group A: symptoms persisted for a single monitoring examination; group B: symptoms persisted for two monitoring examinations; group C: symptoms persisted for all three examinations; group D: none persisted. The occurrence rates of postoperative residual symptoms in groups $\mathrm{A}$ and $\mathrm{C}$ on the first day after surgery and in group $\mathrm{C}$ on the day of discharge were higher than that in group $\mathrm{D}$ $(\mathrm{P}<0.001)$.

\section{Discussion}

The BR can be used as a routine monitoring method during facial nerve microvascular decompression, which is useful when the LSR cannot be elicited or disappears prematurely mid surgery. The BR also has definite specific manifestations of disappearance after vascular decompression similar to the LSR (3). In this study, we investigated whether the use of the BR in HFS surgery is comparable to that of the LSR, and explored its significance for guiding IONM. Furthermore, the combined application of monitoring technology plays a guiding role in judging the compression vessel and location. The disappearance of abnormal response of LSR and BR is the evidence of facial nerve decompression. Some studies show that patients with disappearance of LSR after decompression have a better prognosis in the long-term follow-up period of more than 2 years (7).

In our study, the occurrence rate of the BR reached $88.98 \%$, which was lower than that of the LSR. This may be due to limitations of the technologies used for different monitoring techniques. In this case, it would be advantageous for future research to focus on eliminating these errors in order to provide more accurate statistical analysis. If only one monitoring method is implemented, the BR is not the recommended choice. Instead, the MARLSR is the primary recommendation, followed by the ZYGLSR. If two monitoring methods are to be implemented, the strongest recommendation is for combined application of the MAR-LSR and ZYG-LSR. The non-occurrence rate of the BR was comparable to the combined application of the three methods. There will remain $0.85 \%$ of cases where none of the three monitoring methods are viable.

The disappearance rate of the BR was comparable to that of the MAR-LSR, while that of the ZYG-LSR was relatively low. This suggests that if the BR can produce abnormal motor responses, the monitoring applications are similar for the MAR-LSR, making it suitable for intraoperative monitoring.

It has been established that the BR is a cutaneous reflex which represents a highly organized and useful mechanism in humans $(4,13,14)$. Its nerve conduction pathway contains one simple and one complicated reflex (Figure 2).

R2 and R2' are elicited through a polysynaptic pathway, which is difficult to study during general anesthesia surgery. Therefore, in this study, we used the directly stimulated R1 


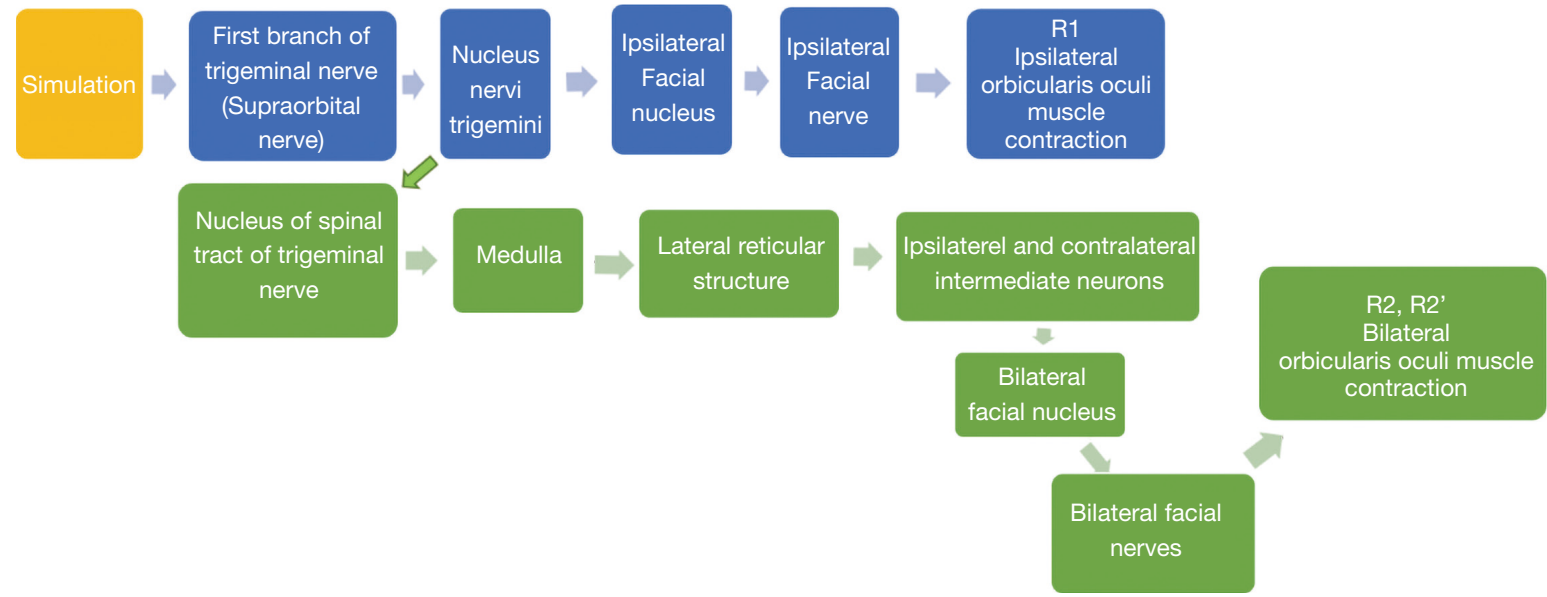

Figure 2 BR nerve conduction pathway. BR, blink reflex.
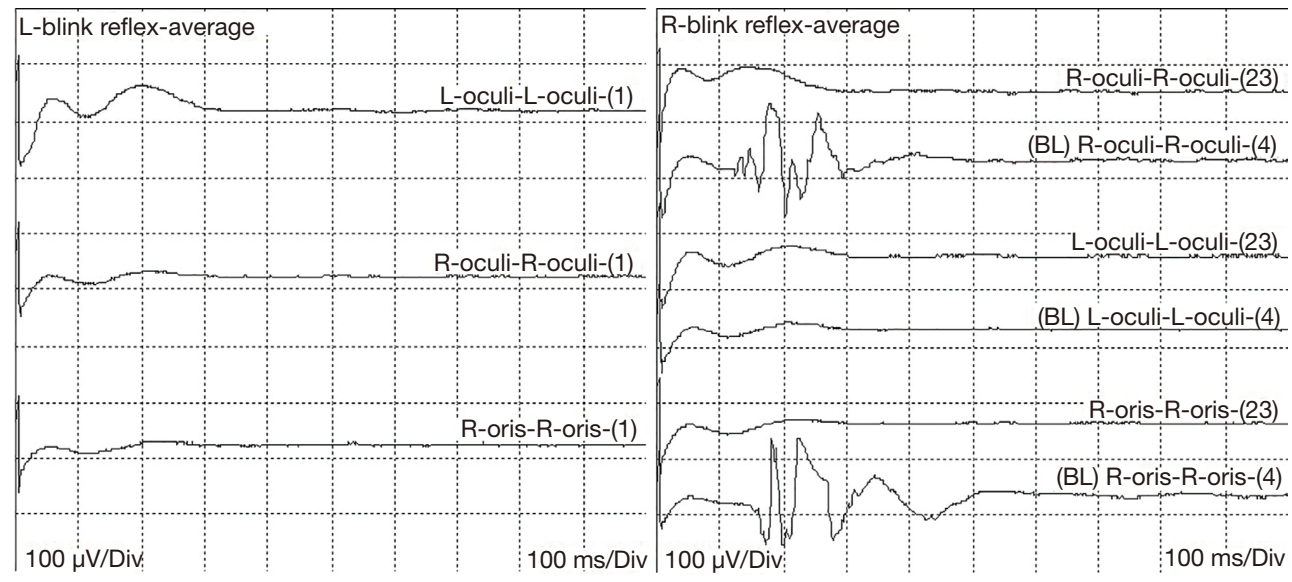

L-oculi: left orbicularis oculi muscle, R-oculi: right orbicularis oculi muscle; R-oris: right orbicularis oris muscle

Figure 3 Specificity of abnormal motor potential elicited by the BR. (A) BR reaction on the non-affected side, and (B) BR reaction on the affected side, showing abnormal motor potential in R-oris. BR, blink reflex.

wave to represent the $\mathrm{BR}$, and observed abnormal motor potentials from the muscle action potential generated by the orbicularis oris. The BR allows the monitoring of the specific pathway as the corresponding orbicularis oris muscle action potential is never elicited in the contralateral trigeminal nerve during surgery (Figure 3).

Some researchers have debated whether the formation of the LSR is the F-wave of the facial nerve or a trigeminal reflex $(15,16)$. The waveform analysis in this study shows that the BR latency and amplitude are not the same as the abnormal motor potential generated by the LSR. The BR is a classical trigeminal reflex, and we therefore do not consider the formation of the LSR a trigeminal reflex. As a result, the pathophysiological mechanism of HFS is more likely to be the enhanced excitability of the facial motor nucleus. The elevated motor neuron excitability and differential effects of desflurane between the spasm and nonspasm sides support a mechanism of central pathophysiology in HFS (17). Monitoring of facial muscle motor evoked potentials during microvascular decompression for HFS shows that the development of HFS in and its alleviation with MVD are related to changes in facial motor nucleus activity (18).

We found no statistical difference in the stimulation currents required to elicit the three reflexes, suggesting that the average occurrence current is similar for each monitor. 
Some researchers have also suggested that the stimulation threshold may reflect the severity of the HFS $(17,19)$. We will continue to study this further in future research.

As can be seen from Figure 2, R1 is formed through the affected lateral nerve nucleus to generate contraction of the orbicularis oculi muscle. Abnormal motor potentials in the orbicularis oris muscle may be related to a decrease in the excitation threshold of the facial nerve or facial nerve nucleus. By comparing the stimulation threshold of motor evoked potentials from the facial nerve between the affected side and the monitored facial nerve, it has been found that the average threshold evoked potential in the spastic side is significantly lower than that in the non-spastic side (16-18).

Patients in the present study monitored during surgery still had positive reactions after the BR, MAR-LSR, and ZYG-LSR, which is consistent with previous reports (20). In a multicenter statistical analysis, around $30-50 \%$ of patients gained no relief of their symptoms (20-22). In our study, these patients made up only $26.1 \%$ of the study sample. There were also fewer residual symptoms in our study, which could have been due to the surgeon, the joint monitoring system, and close attention. We followed up the patients 90, 180 and 360 days after operation. Among them, 34 were lost, all from group D. there were still 2 patients with facial spasm in group A, but the attack frequency and degree were significantly improved; One patient in group $\mathrm{B}$ had symptoms, and the symptoms were completely relieved after about half a year; In group C, there were 2 symptomatic patients, of which 1 was completely relieved after 1 year, and 1 was still residual, but the attack frequency and degree were less than those before operation; Five symptomatic patients in group D showed complete remission at 90 day follow-up, ranging from 1 week to 2 months after discharge.

\section{Conclusions}

The BR, as a routine HFS examination item, was found to be suitable for intraoperative monitoring combined with the LSR based on the long-term practice by the authors. It can be used as a neuroelectric conduction research method for the pathophysiology of facial spasm. An increase in the threshold of the facial nerve or facial nerve nucleus may produce the corresponding symptoms. It also helps the neurophysiologic intraoperative monitors to increase the rate of LSR disappearance, and reduces residual symptoms after surgery. To some extent, this may reflect the integrity of the trigeminal reflex during surgery.

\section{Acknowledgments}

Funding: The present study was supported by the Science and Technology Planning Project of Guangzhou (grant No. 201804010394) and Medical Scientific Research Foundation of Guangdong Province (grant No. A2019399).

\section{Footnote}

Reporting Checklist: The authors have completed the STROBE reporting checklist. Available at https://dx.doi. org/10.21037/apm-21-3197

Data Sharing Statement: Available at https://dx.doi. org/10.21037/apm-21-3197

Conflicts of Interest: All authors have completed the ICMJE uniform disclosure form (available at https://dx.doi. org/10.21037/apm-21-3197). The authors have no conflicts of interest to declare.

Ethical Statement: The authors are accountable for all aspects of the work in ensuring that questions related to the accuracy or integrity of any part of the work are appropriately investigated and resolved. All procedures performed in this study involving human participants were in accordance with the Declaration of Helsinki (as revised in 2013). This study was approved by the Medical Ethics Committee of Guangdong Second Provincial General Hospital (DG2H-KY IRB-AFSC.10-01.1). All patients provided their consent for inclusion in the study.

Open Access Statement: This is an Open Access article distributed in accordance with the Creative Commons Attribution-NonCommercial-NoDerivs 4.0 International License (CC BY-NC-ND 4.0), which permits the noncommercial replication and distribution of the article with the strict proviso that no changes or edits are made and the original work is properly cited (including links to both the formal publication through the relevant DOI and the license). See: https://creativecommons.org/licenses/by-nc-nd/4.0/.

\section{References}

1. Study Group of Functional Neurosurgery in Chinese Neurosurgical Society; Committee of Functional Neurosurgical Experts in Chinese Congress of Neurological Surgeons. A Chinese experts consensus 
of neurophysiological evaluating for microvascular decompression. Zhonghua Wai Ke Za Zhi 2017;55:725-33.

2. Son BC, Ko HC, Choi JG. Intraoperative monitoring of $\mathrm{Z}$-L response (ZLR) and abnormal muscle response (AMR) during microvascular decompression for hemifacial spasm. Interpreting the role of ZLR. Acta Neurochir (Wien) 2018;160:963-70.

3. Choi SI, Kim MW, Park DY, et al. Electrophysiologic investigation during facial motor neuron suppression in patients with hemifacial spasm: possible pathophysiology of hemifacial spasm: a pilot study. Ann Rehabil Med 2013;37:839-47.

4. Frigeni B, Bivona R, Foresti C, et al. Predictive value of preoperative and intraoperative neurophysiology in evaluating long-term facial function outcome in acoustic neuroma surgery. Otol Neurotol 2020;41:530-6.

5. Jiang $\mathrm{C}, \mathrm{Xu} \mathrm{W}$, Dai $\mathrm{Y}$, et al. Early permanent disappearance of abnormal muscle response during microvascular decompression for hemifacial spasm: a retrospective clinical study. Neurosurg Rev 2017;40:479-84.

6. Zhu W, Sun C, Zhang Y, et al. AMR monitoring in microvascular decompression for hemifacial spasm: 115 cases report. J Clin Neurosci 2020;73:187-94.

7. Kang MC, Choi YS, Choi HK, et al. Efficacy of the disappearance of lateral spread response before and after microvascular decompression for predicting the longterm results of hemifacial spasm over two years. J Korean Neurosurg Soc 2012;52:372-6.

8. Koht A, Sloan TB, Toleikis JR. editors. Monitoring the nervous system for anesthesiologists and other health care professionals. New York: Springer, 2012.

9. Lee S, Park SK, Lee JA, et al. A new method for monitoring abnormal muscle response in hemifacial spasm: a prospective study. Clin Neurophysiol 2018;129:1490-5.

10. Rosenstengel C, Matthes M, Baldauf J, et al. Hemifacial spasm: conservative and surgical treatment options. Dtsch Arztebl Int 2012;109:667-73.

11. Kim JY, Jung S, Song TW, et al. The cornerstone technique of microvascular decompression for hemifacial spasm with vertebral artery offender. World Neurosurg 2019;126:e94-e100.

12. Li F, Liu R. Clinical analysis of microvascular decompression in patients with hemifacial spasm: a retrospective study. Ann Palliat Med 2020;9:318-23.

13. Shahani B. The human blink reflex. J Neurol Neurosurg Psychiatry 1970;33:792-800.
14. Valls-Sole J. Spontaneous, voluntary, and reflex blinking in clinical practice. J Clin Neurophysiol 2019;36:415-21.

15. Misawa S, Kuwabara S, Ogawara K, et al. Abnormal muscle responses in hemifacial spasm: $\mathrm{F}$ waves or trigeminal reflexes? J Neurol Neurosurg Psychiatry 2006;77:216-8.

16. Ishikawa M, Ohira T, Namiki J, et al. Electrophysiological investigation of hemifacial spasm after microvascular decompression: $\mathrm{F}$ waves of the facial muscles, blink reflexes, and abnormal muscle responses. J Neurosurg 1997;86:654-61.

17. Wilkinson MF, Chowdhury T, Mutch WA, et al. Analysis of facial motor evoked potentials for assessing a central mechanism in hemifacial spasm. J Neurosurg 2017;126:379-85.

18. Wilkinson MF, Kaufmann AM. Monitoring of facial muscle motor evoked potentials during microvascular decompression for hemifacial spasm: evidence of changes in motor neuron excitability. J Neurosurg 2005;103:64-9.

19. Feroze RA, McDowell MM, Balzer J, et al. Estimation of intraoperative stimulation threshold of the facial nerve in patients undergoing microvascular decompression. J Neurol Surg B Skull Base 2019;80:599-603.

20. von Eckardstein K, Harper C, Castner M, et al. The significance of intraoperative electromyographic "lateral spread" in predicting outcome of microvascular decompression for hemifacial spasm. J Neurol Surg B Skull Base 2014;75:198-203.

21. Tobishima H, Hatayama T, Ohkuma H. Relation between the persistence of an abnormal muscle response and the long-term clinical course after microvascular decompression for hemifacial spasm. Neurol Med Chir (Tokyo) 2014;54:474-82.

22. Song H, Xu S, Fan X, et al. Prognostic value of lateral spread response during microvascular decompression for hemifacial spasm. J Int Med Res 2019;47:6120-8.

(English Language Editor: C. Betlazar-Maseh)

Cite this article as: Zhang $\mathrm{Y}, \mathrm{Xia} \mathrm{L}$, Lin J, Zhang $\mathrm{Y}$. Retrospective study of the combined application of the blink reflex and lateral spread reflex in microvascular decompression of facial nerve surgery. Ann Palliat Med 2021;10(12):12310-12318. doi: 10.21037/apm-21-3197 